\title{
Development of a machine vision system for rice seed inspection system
}

\author{
1,2,3* Ruslan, R., ${ }^{1}$ Khairunniza-Bejo, S., ${ }^{2}$ Rukunudin, I.H, ${ }^{1}$ Jahari, M. and ${ }^{3}$ Ibrahim, M.F. \\ ${ }^{1}$ Department of Biological and Agricultural Engineering, Universiti Putra Malaysia, UPM 43400 Serdang, \\ Selangor Malaysia \\ ${ }^{2}$ School of Bioprocess Engineering, Universiti Malaysia Perlis, UniMAP 02600 Arau, Perlis Malaysia \\ ${ }^{3}$ Center of Excellence for Advanced Sensor Technology (CEASTech) Universiti Malaysia Perlis, UniMAP \\ 02600 Arau, Perlis Malaysia
}

\begin{abstract}
Article history:
Received: 19 May 2020

Received in revised form: 15 September 2020

Accepted: 30 December 2020

Available Online: 31

December 2020
\end{abstract}

Keywords:

Machine vision,

Weedy rice,

Machine learning,

Image acquisition,

Image processing,

Classification

DOI:

https://doi.org/10.26656/fr.2017.4(S6).049

\begin{abstract}
Rice seed production in Malaysia is greatly dependent on the purity of the cultivated paddy seed produced through the government certified paddy seed program. The seeds to be marketed by the seed processors must undergo quality control protocol where the seed lots are sampled from the seed farms and seed processing plants for purity analysis by the enforcing agency at the Seed Testing Laboratory of the Department of Agriculture (DoA). The current inspection conducted by the laboratory is based on a manual process, which is laborious and time-consuming. Therefore, a prototype (Patent ID: PI2018500018) of a machine vision-based rice seed inspection system (RiSe-IViS) was developed to explore the possibility of replacing the existing manual method in distinguishing the weedy rice and cultivated rice seeds under the Standard Jabatan Pertanian Malaysia (SJPM) standard protocol with a modern, effective and efficient technique using an image processing approach. The developed RiSe-IViS prototype consists of two parts i) hardware configuration and ii) software development. This paper discussed the criteria to be established, challenges and limitation encountered in developing the hardware prototype involving the image acquisition setup, lighting configuration and seed plate design. The importance of each criterion to ensure its reproducibility are also discussed. A software programme was developed to assist the user for image acquisition and analysis. The image processing steps undertaken in the programme are also discussed. The RiSe-IViS is expected to classify major rice seed varieties available in Malaysia against the weedy rice variants with superior accuracy.
\end{abstract}

\section{Introduction}

Machine vision technology has been utilized in several applications in the agricultural sectors, such as land-based and aerial-based remote sensing for the application in precision agriculture, natural resources assessment, fresh produce quality assessment, sorting and classification, and in process automation. Machine vision system developed using colour camera (monochrome or RGB colour camera) are capable of recognizing two-dimensional data attributes (pixel size) in the image such as shape, size, colour and texture in the visible colour region (Chen et al., 2002). In a recent development, machine vision equipped with hyperspectral, near infrared and infrared camera are able to inspect the internal quality of produce under the light invisible to humans such as ultraviolet (UV), near infrared (NIR) and infrared (IR). The use of machine vision techniques offers numerous potential to helps farmers to make a better management decision besides reducing the time taken to solve complex agricultural problems. As for an instance, machine vision equipped with sensing elements and machine learning techniques provides a powerful set of tools that applied for different field application in agricultural practices (Rehman et al., 2019). The systems developed are fairly accurate, nondestructive and yield consistent thus remove the possibility of human error and reduce the time taken for decision making.

Developing a machine vision in the agricultural field poses a challenging task especially in meeting the requirement of the subject for image acquisition. The task can be easy if the subject to be imaged is fairly 
simple and presented in a controlled environment. However, its application in solving the agricultural problems is challenging due to several factors, among others, the uncontrolled lighting in the field, overlapping and touching samples, irrelevant data in the background image and often time, the presence of higher variability in the agricultural object. Selection of the appropriate camera and lens is determined by the inspection task. Suitable resolution and sensor size, depending on the type of camera used is very important in developing a workable machine vision system. The distance of the object to the camera influences the details of the image to be captured. Homogeneous lighting system plays an important role to illuminate the object consistently for image acquisition while correct lighting selection highly influences the data captured in an image.

The application of machine vision system in agricultural sectors related to grain industry mainly focused in varietal classification (Shahin and Symons, 2001; Granitto et al., 2002; Pazoki et al., 2014; Golpour et al., 2014; Chaugule and Mali, 2014; Anami et al., 2015; Huang and Chien, 2017), grading application (Tanabata et al., 2012; Kaur and Singh, 2013; Hanibah et al., 2014; Azman et al., 2014; Aznan et al., 2016; Khairunniza-Bejo et al., 2016), moisture content estimation (Farid et al., 2014), seed germination (Lurstwut and Pornpanomchai, 2016) and disease and pest detection (Khairunniza-Bejo and Jamil, 2013; Zhang et al., 2017; Shi et al. 2017). In rice industry, its application in grading has proven effective in detecting fissures in rice kernel (Lan et al., 2002), defects detection in rice such as germs, disease, glumes (Cheng and Ying, 2005) and husk (Jamil and Khairunniza-Bejo, 2014); and detection of immature paddy seed (Teoh and Bakar, 2009; Khairunniza-Bejo and Sudin, 2014; Azman et al., 2014).

In the context of the rice industry in Malaysia, detection of weedy rice within the cultivated rice seed is vital due to its threat to the yield of rice during field production. Currently, rice seed testing requires laboratory workers to count, identify and distinguish between the cultivated rice seed and weedy rice seeds or off-type seeds. There is no machine vision system ever developed to address the problem of weedy rice contamination in the rice seed processing plant. Therefore, this research aimed to explore the possibility of replacing this manual process with a machine vision system to reduce the time taken for inspection and human error due to tiredness. Specifically, the emphasis of this project is to develop a laboratory-scale image recognition system that can identify weedy rice among the cultivated rice seed based on seed variety. The one-to -one model between weedy rice and cultivated rice seed variety is to be developed since the application is varietal based classification. Criterions used in developing the hardware prototype involving the image acquisition setup, lighting configuration, seed plate design and overall setup to make a workable machine vision system using an area scan camera will also be discussed in this paper.

\section{Methodology}

This research aimed to develop a machine vision prototype for weedy rice seed identification within local Malaysian cultivated rice seeds varieties using image processing and classification technique. The research gap lies in the development of the machine vision itself including the hardware setup for image acquisition, finding the right illumination module, specifying the distance between object and camera, background selection for seed holder, image processing and analysis program.

\subsection{Selection of camera and optic lens}

Selection of correct lenses and optics is the most important task to match the image sensor size. Required resolution of the camera can only be obtained by choosing the correct pairs of the lens. If a low-resolution lens is paired with a high-resolution image sensor, the overall machine vision system becomes limited by the resolution of the lens. As part of the image acquisition system, various size of image sensor area scan camera and lens with different specification were tested. The most important specification for the area scan camera was the resolution and pixel size because it indicates the camera ability to provide high information on a small seed image. A complementary metal oxide semiconductor (CMOS) sensor was chosen over charge couple device (CCD) sensor due to its lower cost and improved quality as near to CCD sensor.

For this research, the design constraint was established prior to the hardware selection. A maximum height of the overall system was set to not exceed $1 \mathrm{~m}$. Therefore, the working distance from the front of the lens to the seed kernel on the seed plate was limited between $10-20 \mathrm{~cm}$. A sharp image is required for robust inspection and thus, narrow down the selection of the lens focal length. The longer the focal length, the narrower the angle of view, the higher the magnification. Figure 1 depicts the effect of focal length and working distance on the seed sample. It shows an image plane at a certain focal length and seed samples at a distance of $\mathrm{H}_{1}$, the low number of seeds image were viewed, due to higher magnification of the image size and smaller field 
of view (FOV). At distance $\mathrm{H}_{1}$, one single seed kernel consists of high pixels number. Meanwhile, at distance $\mathrm{H}_{2}$, the higher number of seeds image, due to bigger FOV but the lower magnification of the image size. At distance $\mathrm{H}_{2}$ one single seed kernel consists of low pixels number. Thus, for lens selection, it is important to balance the focal length with the desired clarity at a given specified distance from the seed samples. An entocentric lens with fixed focal length $25 \mathrm{~mm}$ with 6 MP is paired to the 6MP CMOS area scan camera with a sensor size of $7.4 \mathrm{~mm} \mathrm{X} 4.9 \mathrm{~mm}$ at $13.7 \mathrm{~mm}$ working distance for this project. At this distance, a shallow depth of field is maintained by sharply focusing more on the seed sample rather than the background. To allow shallow depth of field, large aperture (low F-stop) was used for the iris opening and adjusting the exposure time to $2000 \mu \mathrm{s}$ for colour image acquisition. For the monochrome image, the black level was adjusted to 200 to influence the image contrast due to low F-stop number. Figure 2 shows the effect of clarity on different working distance with a fixed focal length using the selected area scan camera. Clarity is defined, as the image captured is able to see visible hair protrusion on the seed kernel.

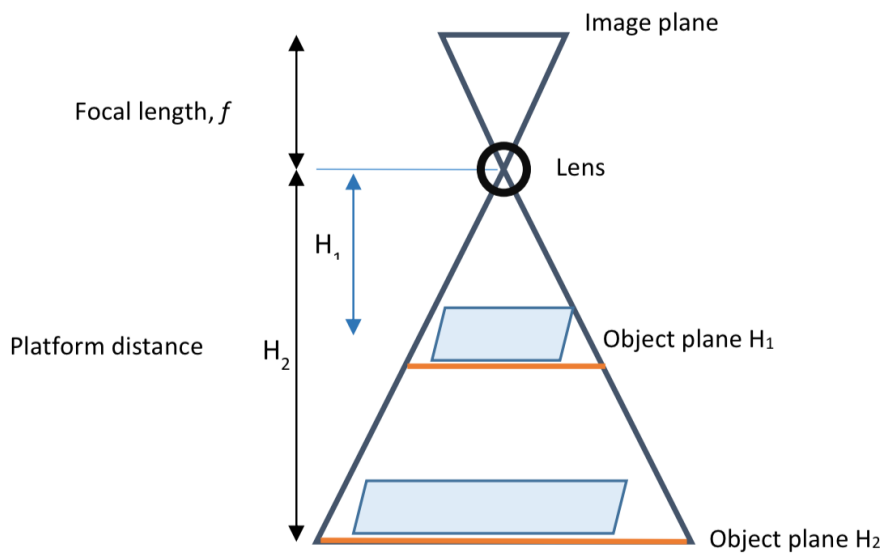

Figure 1. The effect of focal length and platform distance on the magnification of the image plane. (a)

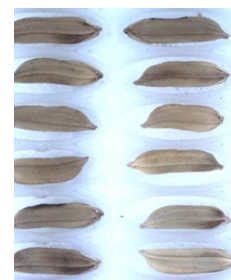

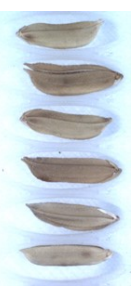

(b)

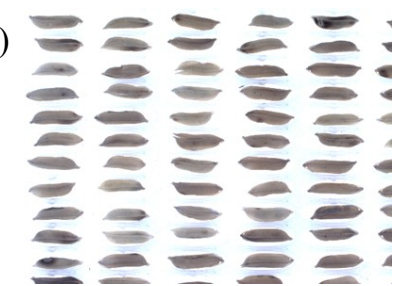

Figure 2. Image acquired using simple LED configuration taken from different working distance a) $14 \mathrm{~cm} \mathrm{b)} 23 \mathrm{~cm}$.

To determine each of the seed kernel measurement, object size is calculated using the sensor information such as number of pixel count, pixel size working distance and the focal length of the lens as shown in Equation 1. This measurement is important in calculating the real value of morphological features of the seed kernel for image analysis and classification. object size $=\frac{\text { (number of pixel count } \times \text { pixel size } \times \text { working distance) }}{\text { focal length }}$

Table 1 shows the specification of the selected area scan camera while Table 2 shows the choices of the lens with the specification of 6MP. The focal length of the lens varied from $8 \mathrm{~mm}$ to $50 \mathrm{~mm}$. Different focal length requires a different working distance. The various combination between the camera and lens were tested, however, the best lens with the focal length of $25 \mathrm{~mm}$ was chosen to be used in this project with adequate working distance. The working distance and image clarity are at its best with the limitation on the height of the overall imaging system.

Table 1. Area scan camera specification

\begin{tabular}{lll}
\hline No & Camera Specification & MVCA060-10GC, HIK Vision \\
\hline 1 & Sensor type & CMOS \\
2 & Max image circle & $1 / 1.8 "$ \\
3 & Sensor size & $7.9 \mathrm{~mm}$ diagonal \\
4 & Resolution & $6 \mathrm{MP}$ \\
5 & H x V & $3072 \mathrm{px} \times 2048 \mathrm{px}$ \\
6 & Pixel size & $2.4 \mu \mathrm{m} \times 2.4 \mu \mathrm{m}$ \\
\hline
\end{tabular}

\subsection{Selection of lighting}

Various lighting effect as shown in Figure 3 was experimented before selecting the correct light for image acquisition. A normal LED bulb with $10 \mathrm{Watt}$ at a $6500 \mathrm{k}$ temperature that provides a bright amount of blueish hue similar to daylight bulb was tested. However, the normal LED bulb did not provide enough brightness and uniform illumination on the FOV, thus the shadow exhibits inside the seed holes. The LED bulb was not diffused as intended to illuminate the sample area, therefore other alternatives were sought. To overcome the uneven illumination, a low angled diffused square illumination (DLW2-60-070-1W-24V) industrial LED lighting was tested as a front light and it proved to provide uniform illumination on the seed samples. Shadows were eliminated and the module used a single channel lighting controller with brightness range from 0 10. Another problem occurred that the image using only a front light will have a background of the seed holder. A high-intensity backlight (BHLX3-00-320x320-X-W$24 \mathrm{~V}$ ) was coupled together as part of the illumination (a)

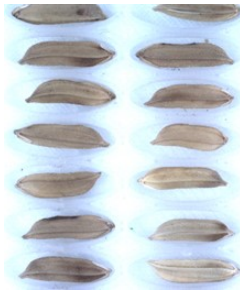

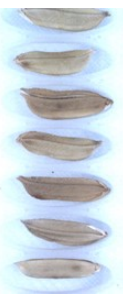

(b)

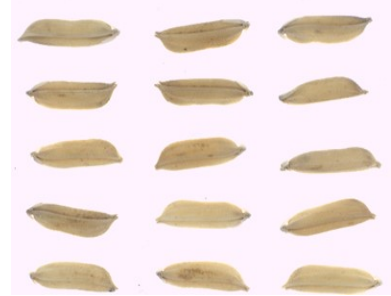

Figure 3. Different lighting setup exhibits different effect on the image a) image used normal LED bulb on the front and back of the sample b) image used an industrial LED lighting, front light and backlight 
Table 2. Various lens specification

\begin{tabular}{|c|c|c|c|c|c|c|}
\hline \multirow[t]{2}{*}{ No } & \multirow[t]{2}{*}{ Lens Specification } & $\begin{array}{c}\text { MVL-HF0828M- } \\
\text { 6MP }\end{array}$ & $\begin{array}{c}\text { MVL-HF1628M- } \\
\text { 6MP } \\
\end{array}$ & $\begin{array}{c}\text { MVL-HF2528M- } \\
\text { 6MP }\end{array}$ & $\begin{array}{c}\text { MVL-HF3528M- } \\
\text { 6MP } \\
\end{array}$ & $\begin{array}{c}\text { MVL-HF5028M- } \\
\text { 6MP } \\
\end{array}$ \\
\hline & & \multicolumn{5}{|c|}{ Fix focal manual iris 6MP } \\
\hline 1 & Focal length & $8 \mathrm{~mm}$ & $12 \mathrm{~mm}$ & $25 \mathrm{~mm}$ & $35 \mathrm{~mm}$ & $50 \mathrm{~mm}$ \\
\hline 2 & f/No & & & $\mathrm{f} / 2.8$ & & \\
\hline \multirow{2}{*}{3} & \multirow{2}{*}{ Maximum image format } & & & $1 / 1.8 ”$ & & \\
\hline & & & & $9 \mathrm{~mm}$ diameter & & \\
\hline \multirow[t]{3}{*}{4} & Field of View D & $58.5^{\circ}$ & $41.2^{\circ}$ & $19.8^{\circ}$ & $13.8^{\circ}$ & $9.7^{\circ}$ \\
\hline & $\mathrm{H}$ & $49.3^{\circ}$ & $34.4^{\circ}$ & $16.3^{\circ}$ & $11.3^{\circ}$ & $8.0^{\circ}$ \\
\hline & $\mathrm{V}$ & $34^{\circ}$ & $23.4^{\circ}$ & $10.9^{\circ}$ & $7.6^{\circ}$ & $5.4^{\circ}$ \\
\hline
\end{tabular}

Table 3. Industrial light specification

1 Image

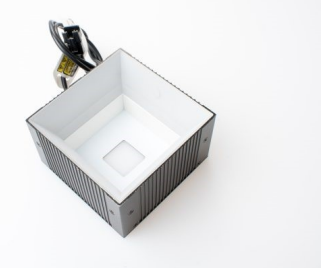

LED

3 Casing material

4 Storage temperature range

5 Weight

6 Length/outer diameter

7 Width/ inner diameter

8 Thickness/ height
Aluminium

Temp 0-45, Humidity $20-85 \%$ $300 \mathrm{~g}$

$76.2 \mathrm{~mm}$

$40.0 \mathrm{~mm}$

$45 \mathrm{~mm}$

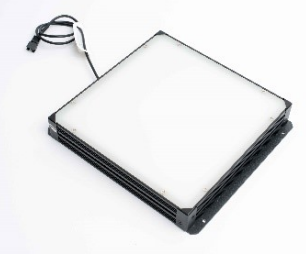

LED

Aluminium

Temp 0-45, Humidity $20-85 \%$

$1900 \mathrm{~g}$

$356 \mathrm{~mm}$

$338 \mathrm{~mm}$

$30 \mathrm{~mm}$ system. The uniform diffused light from backlight penetrates the white seed plate thus, remove the background of the seed image instantaneously. With this combination, the program developed for image processing become easier as only simple thresholding in removing the white pixel value is needed. The lighting specifications used in this project are listed in Table 3.

\subsection{Seed holder design}

Several factors influenced the seed plate design for this system. Seed plate size, number of holes and the dimension of the holes, background colour and the effect of the background on the image. Various designs were tested with different background and final design are as presented in Figure 4. The effect of different background colours was discussed in Ruslan et al. (2018).

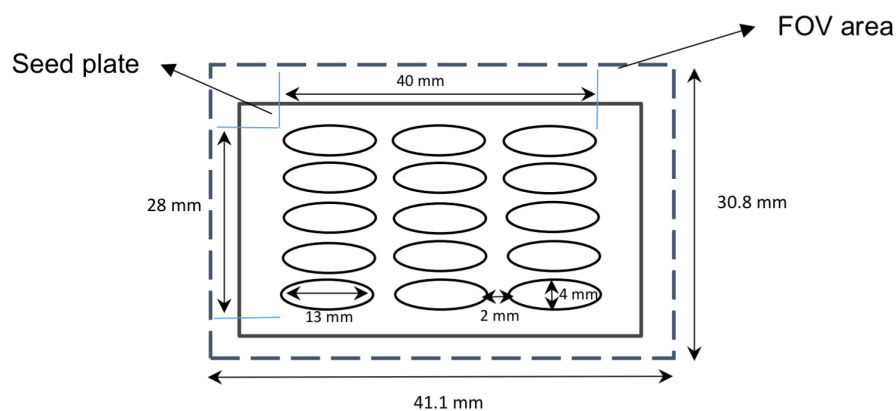

Figure 4. Seed holder design to fit with specified image FOV
The size of the seed holder was determined from the calculation of the field of view based on the maximum sensor size, focal length and working distance as depicted in Equation 2:

field of view $=$ focal length $\frac{\text { working distance }}{\text { (sensor size-1) }}$

With the selected sensor size of maximum 1/8" (7.2 $\mathrm{mm} \times 5.4 \mathrm{~mm}$ ) and using $25 \mathrm{~mm}$ focal length, at a working distance of $137 \mathrm{~mm}$, the field of view was 41.1 $\mathrm{mm}$ X $30.8 \mathrm{~mm}$. Therefore, the sizing of the seed plate and number of holes in one image was designed to be limited by this FOV. Another limitation to the design of the seed holder is the selection of front light was limited to a size $10 \mathrm{~cm} \times 10 \mathrm{~cm}$ to illuminate the seed sample. To design the seed holes, various varieties of Malaysia longgrain rice seed were tested and later decided to compromise for $13 \mathrm{~mm}$ long and $4 \mathrm{~mm}$ dimension with a circular shape to ensure the seed is non-touching and not

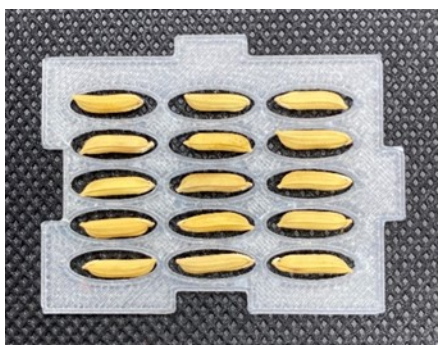

Figure 5. Finalized seed holder with 15 holes per image.

(C) 2020 The Authors. Published by Rynnye Lyan Resources 
overlapped with each other. The background colour of the seed holder was decided to be based on clear filament colour used in the 3D printer as shown in Figure 5.

\section{Results and discussion}

\subsection{Final prototype of the machine vision}

The finalized prototype names as RiSe-IViS (PI2018500018) as in Figure 6 was built using an aluminium angled bar and connected and formed a hard case structure. The body frame of the prototype was based on a 3D printer body casing. The image acquisition and illumination module were installed on the skeleton of the 3D printer. Various connector parts were designed using Solid Works and printed using a 3D printer. The outer case was later built using aluminium sheet and a door was attached to it to allow sample placement. The aluminium body case was covered with stickers to enhance the appearance of the prototype. A platform was built for backlight placement and seed kernel was arranged on the seed plate. The seed holder arranged in $6 \times 4$ seed holder to allow for 360 seeds per run. The arrangement of the seed holders formed a seed plate and was placed on a clear perspex glass and later was slide on top of the backlight LED surface to allow for illumination from the bottom of the sample. The finalized height of the camera system and lighting is depicted in Figure 7. The system was designed with a moving camera attached with front light. The camera took the image of each seed holder and moves from one seed holder to another on the $\mathrm{X}$ and $\mathrm{Y}$ direction. The imaging of the seed samples was done in a close chamber with uniform illumination from the LED lighting to avoid stary light from outside. Image of the seed sample was acquired in two types, monochrome (black and white space) and RGB colour space image. Each image frame consists of 15 seeds. The image was stored as Portable Network Graphics (.png) in a laptop connected to the machine vision using EtherCAT6 cable and later used for analysis.
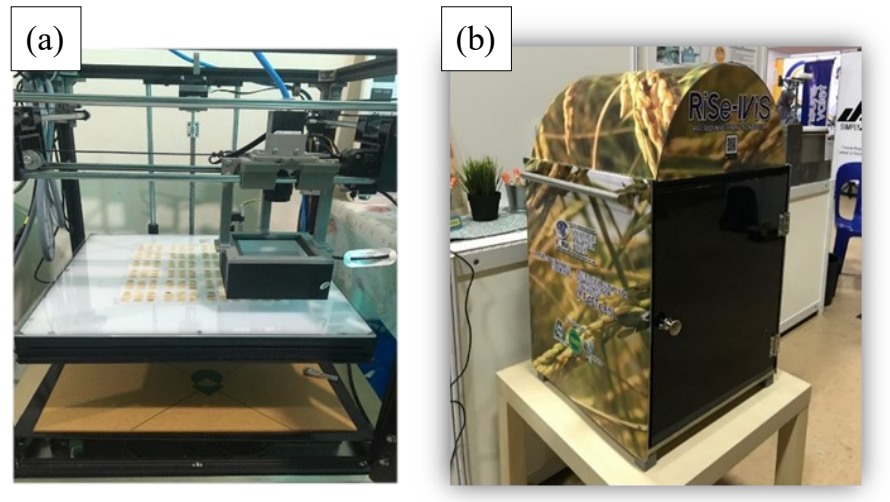

Figure 6. Final design of the machine vision a) internal look of the system assembly b) overall look of the machine vision

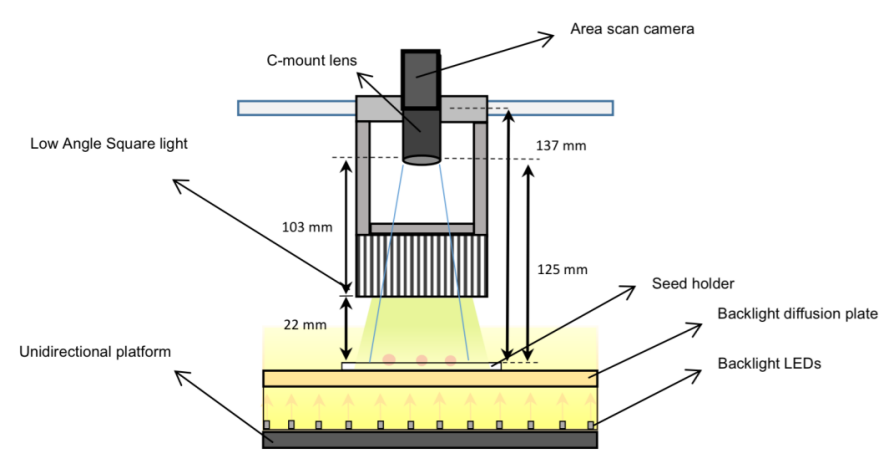

Figure 7. The image acquisition system setting.

The overall design of the machine vision is easy to carry with two long handles on the side of the outer casing. All the components inside the machine vision were secured properly to the casing to ensure no unnecessary movements during transportation. The RiSeIViS is suitable to be placed either on a bench or tabletop near to a power supply.

\subsection{System integration, image processing and analysis}

System integration between hardware and software of the machine vision was integrated with LabView (2016). For image acquisition and data analysis, a graphical user interface (GUI) was developed to assist end-user. In LabView, programming was carried out to acquire an image from the area scan camera. Simple image processing programme was developed for monochrome and RGB images separately. The most important steps in image processing were image segmentation. For RGB image, threshold-based segmentation was employed for each of the colour planes to separate between foreground and background. The selection of thresholding value was defined based on the RGB histogram. For example, in Red colour plane, the pixel value of $0-250$ was retained and converted to binary value 1 (white) in the image segmentation process. Pixel value outside of $0-250$ becomes 0 (black). For monochrome image, simple thresholding on pixel value between $0-3500$ (image format is Mono 12 thus pixel value ranges from 0 - 4600) was converted into binary value 1 while values outside the region become 0 . The resulted image from image segmentation was binary with 1 for the seed and 0 for the background. The pixel value corresponding to 1 was masked to display the seed image in RGB or Mono format. The image thresholding was kept consistent for all images to allow for uniform data extraction. The IMAQ particle measurement counts the particle (pixel) based on the measurement to be extracted using the masked image. The processed images were used for morphological, colour and textural features extraction. The data extracted was saved in an Excel file for future analysis in the machine learning programme. Machine learning algorithm such as neural network, discriminant analyses and other techniques were chosen 
and to be compared in developing the model for variety classification. Preliminary classification results based on morphology features using this prototype was reported in Ruslan et al. (2019). The RiSe-IViS is expected to classify major rice seed varieties available in Malaysia against the weedy rice variants with superior accuracy.

\section{Conclusion}

The machine vision system has great potential in solving agricultural problems. This type of system would become a very important and routine feature in the near future, given the advancement in sensing technology. The current prototype has proved that weedy rice is able to be identified among other cultivated rice seed using image classification technique. This machine vision can be improved by having a LED display panel on the front of the case once the models for the classification is developed, therefore there is no need for laptop connection. With a click of the start button on the panel, the RiSe-IViS could do the imaging and display result instantaneously. The static imaging system is also possible to be improved by converting the platform into a conveyor or chute system for faster image acquisition. Besides using area scan camera and utilizing the visible range, other sensing devices such as hyperspectral, nearinfrared, and multispectral camera are available to be explored. One of the main constraints in developing machine vision is the cost. The selection of the sensing system greatly influenced by the problem to be solved, given there are many choices of methods to be adopted. In near future, developing a machine vision will be costeffective, more accurate and robust. With the advancement in artificial intelligence, a positive future for machine vision is on progress.

\section{References}

Anami, B.S., Naveen, N.M. and Hanamaratti, N.G. (2015). Behavior of HSI Colour Co-Occurrence Features in Variety Recognition from Bulk Paddy Grain Image Samples. International Journal of Signal Processing, Image Processing and Pattern Recognition, 8(4), 19-30. https://doi.org/10.14257/ ijsip.2015.8.4.02

Azman, N., Khairunniza-Bejo, S., Ismail, W.I.W. and Wayayok, W. (2014). Estimating Maturity of Paddy Using RGB Colour Space. Journal of Advanced Agricultural Technologies, 1(2), 119-124. https:// doi.org/10.12720/joaat.1.2.119-124

Aznan, A.A., Rukunudin, I.H., Shakaff, A.Y.M., Ruslan, R., Zakaria, A. and Saad, F.S.A. (2016). The use of machine vision technique to classify cultivated rice seed variety and weedy rice seed variants for the seed industry. International Food Research Journal,
23(Suppl. 1), S31-S35.

Chaugule, A. and Mali, S.N. (2014). Evaluation of Texture and Shape Features for Classification of Four Paddy Varieties. Journal of Engineering, 2014, 617263. https://doi.org/10.1155/2014/617263

Chen, X.R., Chao, K. and Kim, M.S. (2002). Machine vision technology for agricultural application. Computer and Electronics in Agriculture, 36, 173191. https://doi.org/10.1016/S0168-1699(02)00100$\mathrm{X}$

Cheng, F. and Ying, Y.B. (2005). Machine vision system for quality inspection of bulk rice seeds. Optical Sensors and Sensing Systems for Natural Resources and Food Safety and Quality. Proccedings, 5996, 599616. https://doi.org/10.1117/12.629319

Farid, M., Khairunniza-Bejo, S. and Azman, N. (2014). An approach to estimate moisture content of paddy rice via thermal imaging. Journal of Food, Agriculture and Environment, 12(1), 188-191.

Golpour, I., Parian, J.A. and Chayjan, R.A. (2014). Identification and classification of bulk paddy, brown and white rice cultivars with colour features extraction using image analysis and neural network. Czech Journal of Food Sciences, 32(3), 280-287. https://doi.org/10.17221/238/2013-CJFS

Granitto, P.M., Navone, H.D., Verdes, P.F. and Ceccatto, H.A. (2002). Weed seeds identification by machine vision. Computers and Electronics in Agriculture, 33, 91-103. https://doi.org/10.1016/S0168-1699(02) 00004-2

Hanibah, S.S.B., Khairunniza-Bejo, S., Ismail, W.I.W and Wayayok, A. (2014). Determination of physical rice composition using image processing technique. Journal of Food, Agriculture and Environment, 12 (1), 205-209.

Huang, K.Y. and Chien, M.C. (2017). A novel method of identifying paddy seed varieties. Sensors, 17(4), 809. https://doi.org/10.3390/s17040809

Jamil, N. and Khairunniza-Bejo, S. (2014). Husk Detection Using Thermal Imaging Technology. Agriculture and Agricultural Science Procedia, 2, $128-135 . \quad$ https://doi.org/10.1016/ j.aaspro.2014.11.019

Kaur, H. and Singh, B. (2013). Classification and Grading Rice Using Multi-Class SVM. International Journal of Scientific and Research Publications, 3 (4), 1-5.

Khairunniza-Bejo, S. and Jamil, N. (2013). Preliminary Study on Detection of Fungal Infection in Stored Paddy Using Thermal Image. International Proceedings of Chemical, Biological and Environmental Engineering, 60, 19-23. 
Khairunniza-Bejo, S., Azman, N. and Jamil, N. (2016). Paddy Grading using Thermal Imaging Technology. International Food Research Journal, 23(Suppl. 1): S245-S248.

Khairunniza-Bejo, S. and Sudin, N.M. (2014). Mature and Immature Paddy Identification using Image Processing Technique. Journal of Engineering Science and Technology, 9(3), 326-333.

Lurstwut, B. and Pornpanomchai, C. (2016). Application of image processing and computer vision on rice seed germination analysis. International Journal of Applied Engineering Research, 11(9), 6800-6807.

Pazoki, A.R., Farokhi, F. and Pazoki, Z. (2014). Classification of rice grain varieties using two artificial neural networks (mlp and neuro-fuzzy). Journal of Animal and Plant Sciences, 24(1), 336343.

Rehman, T.U., Mahmud, M.S., Chang, Y.K., Jin, J. and Shin, J. (2019). Current and future applications of statistical machine learning algorithms for agricultural machine vision systems. Computers and Electronics in Agriculture, 156 585-605. https:// doi.org/10.1016/j.compag.2018.12.006

Ruslan, R., Khairunniza-Bejo, S., Rukunudin, I.H., Ibrahim, M.F., Aznan, A.A. and Azizan, F.A. (2018). Effect of Background Colour on Rice Seed Image Segmentation using Machine Vision presented at the 2018 International Conference on Computational Approach in Smart Systems Design and Applications (ICASSDA), p. 1-4. Kuching, Sarawak. https://doi.org/10.1109/ ICASSDA.2018.8477614

Ruslan, R., Bejo, S.K., Rukunuddin, I.H. and Aznan A.A. (2019). Selection of Morphological Features in Classifying Weedy Rice and Rice Seed Varieties using Discriminant Function Analysis. IOP Conference Series, Material Science Engineering, 55, 012014. https://doi.org/10.1088/1757899X/557/1/012014

Shahin, M A. and Symons, S.J. (2001). A machine vision system for grading lentils. Canadian Biosystems Engineering/Le Genie Des Biosystems Au Canada, 43, 7.7-7.14.

Shi, Y., Huang, W., Luo, J., Huang, L. and Zhou, X., (2017). Detection and discrimination of pests and diseases in winter wheat based on spectral indices and kernel discriminant analysis. Computer and Electronics in Agriculture, 141, 171-180. https:// doi.org/10.1016/j.compag.2017.07.019

Tanabata, T., Shibaya, T., Hori, K., Ebana, K. and Yano, M. (2012). SmartGrain: High-throughput phenotyping software for measuring seed shape through image analysis. Plant Physiology, 160(4), 1871-1880. https://doi.org/10.1104/pp.112.205120

Teoh, C.C. and Bakar, B.H.A. (2009). Immature paddy quantity determination using image processing and analysis techniques (Penentuan kuantiti padi tidak matang dengan menggunakan teknik pemprosesan dan penganalisisan imej). Journal Tropical Agriculture and Food Science, 37(2), 241-248.

Zhang, J., Wang, N., Yuan, L., Chen, F. and Wu, K. (2017). Discrimination of winter wheat disease and insect stresses using contiuos wavelet features extracted from foliar spectral measurement. Biosystems Engineering 162, 20-29. https:// doi.org/10.1016/j.biosystemseng.2017.07.003 